\title{
PROGRAMAS DE CONSERVACIÓN DE OBRAS EN DISTRITOS DE RIEGO COMO ALTERNATIVA SUSTENTABLE EN LA ADMINISTRACIÓN DEL AGUA DE USO AGRÍCOLA
}

\section{CONSERVATION PROGRAM WORKS AS AN ALTERNATIVE IRRIGATION DISTRICTS IN SUSTAINABLE WATER MANAGEMENT OF AGRICULTURAL USE}

\author{
Víctor Manuel Peinado-Guevara1; Carmen Camacho-Castro2; Deyanira Bernal- \\ Domínguez3; Omar Delgado-Rodríguez4 y Héctor José Peinado-Guevara5 \\ Escuela de Ciencias Económicas y Administrativas ECEA-UAS1. Facultad de Contaduría y Administración, \\ FCA-UAS, Ciudad Universitaria, Culiacán, Sin. México2. Facultad de Contaduría y Administración, FCA-UAS, \\ Ciudad Universitaria, Culiacán, Sin. México3. Instituto Mexicano del Petróleo, Eje Central Lázaro Cárdenas \\ Norte 152, San Bartolo Atepehuacan4. Escuela de Ciencias Económicas y Administrativas ECEA-UAS, Carretera \\ a las Glorias, Guasave, Sinaloa, México5.
}

\section{RESUMEN}

La escasez del agua es un tema de interés mundial puesto que ya está repercutiendo en el desarrollo social. México no está ajeno a esa problemática y siendo que en varias regiones del país se presentan grandes dificultades para suministrar el agua, principalmente para uso agrícola. En Sinaloa, en reiteradas ocasiones, se ha mencionado en los medios de comunicación, que en el distrito de riego 063, situado al norte del Estado, se presentan problemas de escasez de agua, y aun así se tienen dificultades para la conservación del recurso, puesto que más del $49 \%$ del agua que se destina a la agricultura se desperdicia.

Para resolver esta problemática, los productores y las instituciones gubernamentales destinan recursos importantes para la inversión en la conservación del agua, no obstante, los resultados no han sido del todo satisfactorios puesto que el desperdicio es elevado, situación que motiva a estudiar a fondo las

Recibido: 3 abril de 2012. Aceptado: 26 mayo de 2012.

Publicado como NOTA CIENTÍfICA en Ra Ximhai 8(2): 119126.

Edición Especial: Contaminación y Medio Ambiente. principales debilidades que inciden en el uso sustentable del recurso.

La participación del usuario-agricultor en la administración de la infraestructura hidráulica es importante, puesto que además de aportar recursos económicos para la conservación de la red hidráulica, participa en las actividades de construcción y reparación de infraestructura hidráulica, en la planeación y diseño de estrategias para la conservación del agua. Esta situación requiere de un nivel tecnológico e intelectual apropiado, algo que los productores locales carecen y por lo tanto complica el manejo sustentable del recurso.

Palabras claves: Módulos de riego, escasez hídrica, políticas hídricas y láminas de agua.

\section{SUMMARY}

Water scarcity is an issue of worldwide concern since it is already having an impact on social development. Mexico is not an exception to this problem because in several regions of the country are great 
difficulties in supplying water, primarily for agricultural use. In Sinaloa, it had been mentioned repeatedly by the media that in the Irrigation District 063, located in the northern of the state, there are problems of water scarcity, and yet there still exist difficulties in conserving the resource. More than $49 \%$ of the water used for agriculture is wasted.

To resolve this problem, producers and government agencies spend significant resources for investment in water conservation. However, the results have not been entirely satisfactory because the waste is high, a situation that motivates them to study more deeply the main weaknesses that affect sustainable resource use.

Farmer's participation in the administration of water infrastructure is important, as well as providing financial resources for the conservation of water system; and participation in activities of construction and repaired of water infrastructure. Farmer's should also plan and design strategies for water conservation. This situation requires an appropriate level of technology and intellectual, rather than local producers and thus no complicated sustainable resource management. That is what local producers don't have and therefore it complicates the sustainable management of the resource.

Keyword: Irrigation Modules, water scarcity, water policies, and water bodies.

\section{INTRODUCCIÓN}

El agua ha sido uno de los pilares básicos en el desarrollo de la humanidad; las grandes civilizaciones de todos los tiempos han tenido como una de las columnas de su apogeo la cercanía con las fuentes naturales de agua tales como ríos, lagos, entre otros (Cárdenas G. y Cárdenas J., 2009). Este recurso es fundamental para el desarrollo pleno de la sociedad puesto que, aparte del consumo humano, es primordial en la producción de alimentos. Es importante destacar que, del volumen total de agua que existe en el planeta, únicamente el $1 \%$ puede ser consumido por los seres vivos, el $2 \%$ del volumen total se encuentra congelado en los glaciares y en las regiones polares, mientras que el 97\% restante es agua marina. (De la Lanza y García, 2002). Esta situación pone de manifiesto la urgencia de administrar el agua como elemento importante en el desarrollo económico de un mundo globalizado, donde las constantes transformaciones sociales y la intensa demanda del agua, ha impulsado a los gobiernos en diferentes continentes a centrar la atención en la gestión hidráulica; esto como consecuencia de la insuficiencia y deterioro de la infraestructura hídrica, misma que se ha manifestado en muchos países. Por lo tanto, uno de los principales objetivos en las políticas públicas internacional es el uso y la explotación racional de los recursos hídricos como premisa para asegurar la sustentabilidad ambiental y el suministro eficiente a futuras generaciones.

Un aspecto de suma importancia en la administración del agua, es la necesidad de crear los mecanismos de suministros eficientes del recurso, con el fin de contrarrestar el problema de la escasez, la falta de control en el manejo del agua, la falta de infraestructura con alta tecnología para su conservación y los fuertes desperdicios que existen; situación que obliga a los diferentes niveles de gobierno a fortalecer las estrategias públicas para capitalizar a las instituciones privadas y gubernamentales, creando los instrumentos para el suministro del agua y acondicionar los sistemas de riego en los que se logre el máximo aprovechamiento del recurso. Además de la mayor o menor escasez física del recurso, emergen nuevos 
problemas a los que, hasta la fecha, no se ha prestado la atención que merecen: el creciente deterioro de la calidad del agua y la imposibilidad de hacer frente a las enormes inversiones necesarias para aumentar la cantidad disponible de agua y mejorar su calidad (Biswas, 1999). Estas limitantes pueden desencadenar una grave crisis en el suministro del líquido en el contexto mundial. Aunado a esto, más del 60\% de la producción agrícola mundial corresponde a una agricultura que depende exclusivamente del régimen de lluvias, utilizando el agua precipitada antes de que ésta se concentre en zonas superficiales o subterráneas (Klohn y Appelgren, 1999). Así mismo, el desarrollo de una infraestructura de control del agua para la aplicación del riego permite al agricultor obtener seguridad para la cosecha y arriesgarse a invertir, utilizar variedades de alto rendimiento y aplicar tecnología en los procesos de producción; situación que propició que el riego se convirtiera en la clave de una agricultura productiva $\mathrm{y}$ exitosa.

El problema de la escasez del agua ya empieza a generar complicaciones en México, la disponibilidad de agua presenta una desigual distribución regional y estacional que dificulta su aprovechamiento sustentable; en el norte del país, la disponibilidad de agua por habitante alcanza niveles de escasez críticos, mientras que en el centro y en el sur es más abundante. Entre los años 2000 y 2005, la disponibilidad por habitante disminuyó de 4,841 a 4,573 m3/año, y los escenarios estudiados por la CONAGUA, así como las proyecciones del Consejo Nacional de Población, indican que, para el año 2030, la disponibilidad media de agua por habitante se reducirá a 3,705 m3/año (PND, 2007). Agravando la problemática, la demanda de este recurso se incrementa constantemente por los avances tecnológicos en los procesos de producción, principalmente en las zonas del país donde el recurso es más escaso. Es importante destacar que, en el plano agrícola, México es la séptima nación con mayor superficie de riego en el mundo, consumiendo el $76.8 \%$ del agua disponible en la irrigación de 6.5 millones de hectáreas, de las cuales $25 \%$ se abastecen con $19.2 \mathrm{~km} 3$ de aguas subterráneas extraídas de 3,292 plantas de bombeo y 133,745 pozos profundos (PNH, 2007, CONAGUA, 2010b).

La explotación y ordenación de los recursos hídricos, sigue siendo un factor fundamental en los esfuerzos para la búsqueda del crecimiento económico, el desarrollo sustentable y la reducción de la pobreza. De acuerdo con el gobierno federal, la superficie de riego en el país está concesionada en dos grandes bloques: el 46\% del agua es utilizada por 901 mil agricultores en más de 39 mil Unidades de Riego y el 54\% restante se encuentra a cargo de 552 mil usuarios en 82 Distritos de Riego, mismos que funcionan a través de los módulos de riego (CONAGUA, 2010b).

La gestión en la administración del agua en México se desarrolla en tres diferentes ámbitos; en el contexto nacional se plantean las grandes políticas y estrategias asociadas al manejo y preservación del agua; en el contexto regional se particulariza su instrumentación, considerando las características de cada zona del territorio y; en el ámbito local se aplican estas políticas y estrategias para impactar favorablemente el bienestar social, el desarrollo económico y la preservación del medio ambiente. Para lograr lo anterior, la administración del agua se ha dividido en 13 cuencas hidrológicas y estas a su vez en 85 distritos de riegos y 39,492 unidades de riego, en la que se le da la intervención organizada a los usuarios en la 
gestión para el desarrollo de infraestructura, lo que es fundamental para alcanzar los objetivos propuestos en las políticas hídricas (PNH, 2008).

El $88 \%$ del volumen de agua que se emplea en los distritos de riego proviene de fuentes superficiales (ej. presas, ríos, etc.) $y$ el $12 \%$ restante corresponde a aguas subterráneas que se extraen de los acuíferos a través de pozos profundos. En lo relativo a la infraestructura mayor, en el país existen aproximadamente 2,200 presas de almacenamiento que abastecen a los distritos y unidades de riego, de las cuales, alrededor del 35\% tienen más de 40 años de antigüedad, siendo que su vida útil de diseño es de 50 años (PNH, 2008).

En la Región III, Pacífico Norte, existen diez distritos de riego distribuidos en los estados de Sinaloa, Chihuahua, Durando, Zacatecas y Nayarit, de los cuales seis se localizan en el estado de Sinaloa, la superficie bajo riego es de 805,200 hectáreas, con una demanda requerida de 7,617 hm3 que utilizan como fuente de abastecimientos las aguas superficiales en un 93\% (CONAGUA, 2009c).

En el 2010, la CONAGUA informa que Sinaloa está distribuida en 49 módulos de riego organizados en 8 distritos de riego, que amparan una superficie otorgada de siembra de 753,023 hectáreas, donde laboran 81,409 productores, entre ejidatarios y pequeños propietarios (CONAGUA, 2010b). Con respecto a las organizaciones de usuarios en módulos de riego, su colaboración es fundamental y para esto se han integrado en sociedades civiles, con el propósito de participar en los programas existentes para la conservación de la infraestructura hídrica.

Es importante destacar que, con la reforma del artículo 27 de la Constitución Política de
México y la Ley de las Aguas Nacionales en 1989, el gobierno mexicano, a través de la CONAGUA, inició la transferencia de las actividades de operación, conservación, ingeniería de riego y drenaje, así como la administración de los Distritos de Riego a los usuarios, quienes, organizados en asociaciones civiles, se hacen cargo de la infraestructura secundaria e interparcelaria correspondiente a su respectivo módulo (Sánchez, Arteaga, Reyes y Arana, 2001). Partiendode estaley y reforma constitucional, entre las principales funciones del distrito de riego (DR063) y de los módulos de riego adscritos a ella, se destacan las siguientes atribuciones:

- Participar en la administración, construcción, reconstrucción, operación, conservación y mejoramiento de las obras de infraestructura hidro - agrícola, con que cuenta el modulo de riego.

- Celebrar los actos jurídicos necesarios para la correcta administración, construcción, reconstrucción, conservación y la operación de la infraestructura, maquinaria y equipo de la asociación.

- Construir las obras de infraestructura necesaria, para el adecuado aprovechamiento del agua.

- Realizar las obras de conservación y mantenimientos que sean necesarias en la infraestructura hidroagrícola correspondiente al modulo.

- Llevar a cabo un inventario de infraestructura hidroagrícola existente.

Estas atribuciones, supone que permiten generar los medios necesarios para garantizar el suministro del recurso hídrico a los productores.

Este trabajo tiene como objetivo principal realizar un balance técnico-financiero de los presupuestos que se destinan para la conservación de la infraestructura hidráulica en el DR063, partiendo de los principales 
conceptos en que se aplican los recursos económicos como son pozos, plantas de bombeo, red de distribución, red de drenaje, red de caminos y estructuras; y el efecto que genera en la conservación del agua. Para lograr esto se realizan tales tipos de análisis basado en los programas de actividades de los ciclos agrícolas 2007-2008, 20082009 y 2009-2010, para la conservación de obras de distritos de riegos transferidos, específicamente el caso del DR063.

Descripción del área de estudio

Sinaloa es una de las entidades agrícolas más importantes del país; la agricultura se concentra en la llanura costera, donde las precipitaciones son insuficientes para satisfacer las necesidades hídricas de los cultivos. Debido a esto, la mayoría de las tierras cuenta con infraestructura para riego, cuyos principales cultivos de este tipo son: caña de azúcar, maíz, frijol, papa, cártamo, soya, sin ignorar la importancia que representa la producción hortícola en el Estado. Hacia el pie de la sierra, los terrenos sostienen agricultura de temporal, con la cual se produce principalmente sorgo, maíz, garbanzo, pastos, cártamo y mango, entre otros (INEGI, 2011).

Es importante hacer mención, que el DR063 se ubica en la zona centro norte del Estado de Sinaloa, con una superficie agrícola de 112,688 hectáreas que beneficia a 15,676 usuarios, distribuidos en los municipios de Guasave y Sinaloa de Leyva. En la figura 1 se observa el área de estudio, ubicado entre los meridianos $108^{\circ} 18^{\prime} 00^{\prime \prime}$ y $108^{\circ} 42^{\prime} 00^{\prime}$ de longitud Oeste y los paralelos $25^{\circ} 52^{\prime} 00^{\prime}$ ' y $25^{\circ} 37^{\prime} 00^{\prime \prime}$ de latitud Norte, a una altitud promedio de $40 \mathrm{msnm}$ (González y Hansen, 2009).

El clima en la zona de influencia del DR 063 es seco y cálido, con una temperatura

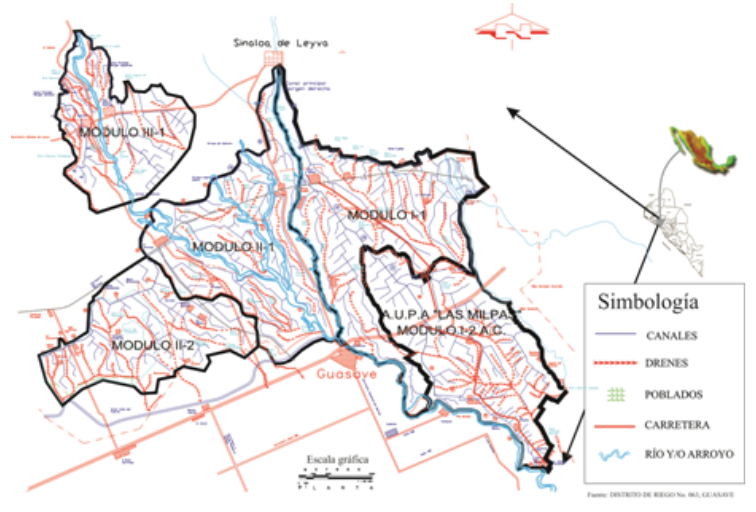

Figura 1. Mapa del distrito de riego 063.

media anual es de $23.9{ }^{\circ} \mathrm{C}$ (temperaturas mínimas y máximas de $4.4{ }^{\circ} \mathrm{C}$ y $42.0{ }^{\circ} \mathrm{C}$, respectivamente) y la precipitación media anual oscila es aproximadamente $409 \mathrm{~mm}$ (González y Hansen, 2009). El DR063 cuenta con dos fuentes de abastecimiento de agua superficial: el Río Sinaloa y el Arroyo Ocoroni. Las aguas del Río Sinaloa son captadas en la presa Gustavo Díaz Ordaz, que abastece una superficie de 102,774 hectáreas de riego; mientras que el Arroyo Ocoroni son captadas en la presa Guillermo Blake Aguilar, abasteciendo una superficie de 9,914 hectáreas (CONAGUA, 2010a). El distrito está comprendido por 5 módulos de riego con una superficie de 112,688 hectáreas y 15,676 usuarios (ver cuadro 1).

\section{MATERIALES Y METODOS}

Para el desarrollo de la presente investigación, se trabajó con los planes y programas de conservación de la infraestructura hidráulica correspondiente al DR063 del estado de Sinaloa, así como de los presupuestos destinados para la lograr tales objetivos. Esto en virtud de que unos de los principales problemas que afectan al sector hidráulico es el alto volumen de agua que se desperdicia y el deterioro que resiente la red de infraestructura hidráulica a nivel 
Diagnóstico del uso y manejo de los residuos de aceite automotriz en el municipio de El

Fuerte, Sinaloa

Cuadro 1. Distribución superficial por módulo de riego en el distrito 063.

\begin{tabular}{lll}
\hline Módulo de riego & Superficie hectáreas & $\begin{array}{l}\text { Número } \\
\text { usuarios }\end{array}$ \\
\hline I-1. Bamoa & 30,514 & 3,933 \\
I-2. Las Milpas & 23,620 & 2,297 \\
II-1. Petatlán & 23,285 & 4,019 \\
II-2. Tetameche & 21,820 & 2,336 \\
III-1. El Sabinal & 9,914 & 1,863 \\
$\begin{array}{l}\text { URDERALES (Unidades de } \\
\text { riego para el desarrollo rural) }\end{array}$ & 3,535 & 1,228 \\
\hline
\end{tabular}

Fuente: elaboración propia, con datos obtenidos en CONAGUA, 2010a.

nacional. Para lograr el objetivo planteado, se contó con la colaboración de personal calificado adscrito a la Comisión Nacional del Agua (CONAGUA), los módulos de riegos adscritos al distrito de riego $063 \mathrm{y}$ de la información proporcionada por la CONAGUA a través del IFAI. Para efecto del presente análisis, se estudiaron los planes y programas de conservación proyectados por los módulos de riego y los presupuestos destinados para ello, esto con el fin de determinar el impacto en el desarrollo de la infraestructura y el beneficio que representa en la búsqueda de la conservación de los recursos hídricos.

\section{RESULTADOS Y DISCUSIÓN}

Ante los fenómenos meteorológicos presentados en febrero de 2011 en Sinaloa, donde las heladas afectaron la mayor parte de la producción agrícola (Fig. 2), y, el plan económico propuesto por los gobiernos locales y federales para la resiembra en los predios dañados, se puso de manifiesto la escasez del agua en el Estado, lo que puede convertirse, a un corto plazo, en un grave problema para el desarrollo del campo.

Esta situación debe motivar a los sectores sociales a concientizar el manejo razonable de los recursos hídricos, pero, sobre todo, generar las condiciones necesarias para la conservación del preciado líquido.
En el análisis de la información obtenida para el desarrollo de la presente investigación en el DR063, se encontró que durante el transporte agua, desde que sale de la presa hasta las parcelas, se establecen tres momentos o instancias. Primero, a nivel de derivadora o red mayor; segundo, de la red mayor se distribuye a los puntos de control de los módulos de riego; y por último, del módulo de riego al usuario o nivel parcela. En esos tres momentos mencionados, existe una fuerte pérdida del vital liquido, es decir, el nivel de eficiencia en la conducción del agua a la derivadora o red mayor, es del $84.5 \%$; de la red mayor al punto de control del distrito de riego, el nivel de eficiencia

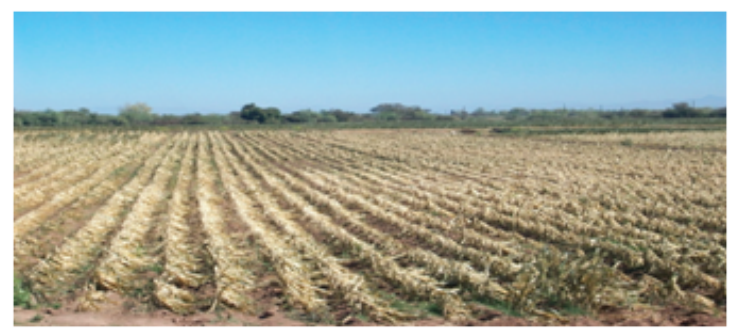

Figura 2. Estragos causados por las heladas atípicas de febrero del 2011en cultivos de maíz en el estado de Sinaloa. Situación similar pudo observarse en el resto de las tierras cultivadas de la región.

en la conducción es del 90\%; y por último, desde el punto de control del módulo de riego a la parcela es del 67\% (CONAGUA, 2008a). Esta situación supone que, de cada $100 \mathrm{~m} 3$ de agua que sale de la presa, solamente se aprovechan $50.95 \mathrm{~m} 3$. Entre los factores que inciden en la pérdida del agua en la conducción a través de la red hidráulica, se encuentran la evaporización y la infiltración pero, sobre todo, el deterioro de la infraestructura hidráulica es el factor más influyente. 
El histograma de la figura 3 corresponde al ciclo agrícola 2009-2010 para el DR063. Cabe señalar que cada módulo de riego dispone de un volumen de agua en función a su extensión territorial, y que los datos reflejan dos valores, un valor de lamina neta, que es la demanda media del agua requerida por una hectárea sembrada; y un valor lamina bruto, que es la cantidad de agua que se debe suministrar para satisfacer la demanda del recurso en la parcela. La diferencia entre estos dos valores, es el volumen de agua que se pierde en la conducción del agua desde el punto de control del módulo de riego hasta la parcela.

De lograr una mayor eficiencia en la conducción del agua y mejorar los sistemas de riegos parcelarios, se puede afirmar que la extensión territorial de uso agrícola susceptible a irrigarse se puede ampliar considerablemente, o bien, ante situaciones de emergencia como las presentadas en febrero de 2011 en la zona de estudio, estar en la posibilidad de garantizar el suministro del agua. Por lo que, para corregir el problema de la pérdida del agua en la conducción, se demandan inversiones millonarias, participando los tres niveles de gobierno, pero sobre todo, la aportación económica de los usuarios. Por otra parte, se deben de

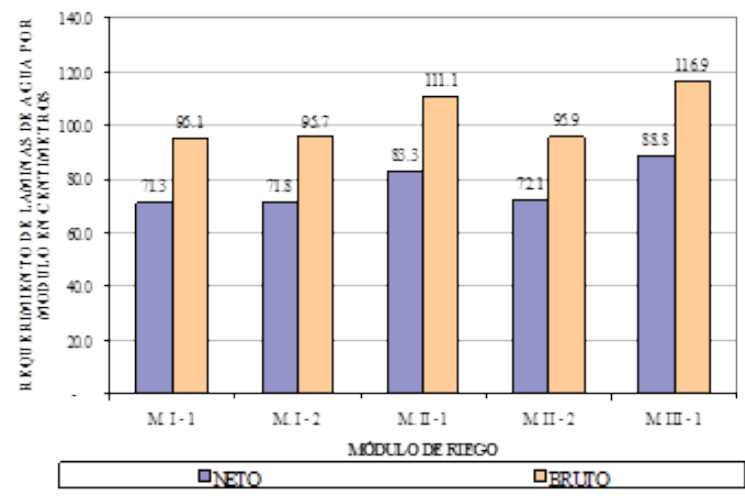

Figura 3. Volúmenes de agua concesionada en el DR 063. Fuente: elaboración propia con datos. establecer los mecanismos para maximizar la conservación del agua y garantizar la administración eficiente del recurso.

Para efectos de profundizar en el tema de la conservación, se realizó un análisis detallado a los planes y programas del DR 063 por módulo de riego en materia de inversión, en los ciclos agrícolas correspondientes del 2007 al 2010.

El estudio comprende la inversión destinada en seis rubros que son: pozos, plantas de bombeo, red de distribución, red de drenaje, red de caminos y estructuras, correspondientes a cada módulo y sus tendencias en los diferentes ciclos agrícolas.

A nivel distrital, la inversión por los ciclos agrícolas 2007 - 2008, 2008 - 2009 y 2009 - 2010 en materia de conservación, es de $\$ 113,329,246.36$, mismo que se detalla en cuadro 2.

En dependencia del estado de la infraestructura hidráulica será el monto invertido en la red de distribución y drenaje.

En el cuadro 3, se observa el comportamiento de los egresos en conservación en los distintos módulos que integran el DR063. Para el análisis se debe considerar la superficie sembrada en función de la inversión aplicada en este rubro. El módulo Bamoa I-1 cuenta con una mayor superficie sembrada con 30,514 hectáreas y el de menor superficie sembrada es el modulo Sabinal III-1 con 9,040 hectáreas, ambas para el ciclo 2009-2010. No obstante, el comportamiento de la inversión depende de la dificultad para acceder al recurso hídrico y no a la superficie irrigada, como se observa en los módulos Petatlán y Bamoa. Por otro lado, se observa que el incremento en las inversiones destinadas a la conservación de 
Diagnóstico del uso y manejo de los residuos de aceite automotriz en el municipio de El

Fuerte, Sinaloa

Cuadro 2. Concentrado de la inversión destinada a la conservación de la infraestructura hidroagrícola del DR 063 en los ciclos agrícolas del 2007 al 2010.

\begin{tabular}{|c|c|c|c|c|c|c|c|c|}
\hline CICLO & CONCEPTO & & $\begin{array}{l}\text { BAMOA I- } \\
1\end{array}$ & $\begin{array}{l}\text { MILPAS } \\
\text { I-2 }\end{array}$ & $\begin{array}{l}\text { PETATLAN } \\
\text { II-1 }\end{array}$ & $\begin{array}{l}\text { TETAMECHE } \\
\text { II-2 }\end{array}$ & $\begin{array}{l}\text { SABINAL } \\
\text { III-1 }\end{array}$ & DR063 \\
\hline \multirow{7}{*}{$\begin{array}{l}2007- \\
2008\end{array}$} & Pozos & & 318,942 & & $1,882,692$ & 190,593 & & $2,392,227$ \\
\hline & $\begin{array}{l}\text { Plantas } \\
\text { bombeo }\end{array}$ & de & - & - & - & 42,634 & - & 42,634 \\
\hline & $\begin{array}{l}\text { Red } \\
\text { distribución }\end{array}$ & de & $3,324,219$ & $3,318,046$ & $3,577,782$ & $2,056,237$ & 587,222 & $12,863,505$ \\
\hline & Red de drenaje & & $2,818,845$ & $2,573,305$ & $2,520,624$ & $3,438,264$ & $1,164,940$ & $12,515,978$ \\
\hline & Red de caminos & & $1,855,855$ & $1,724,740$ & 30,425 & 757,498 & 561,351 & $4,929,869$ \\
\hline & Estructuras & & 479,787 & 248,563 & $1,725,241$ & 343,546 & - & $2,797,137$ \\
\hline & Suma de obra & & $8,797,647$ & $7,864,654$ & $9,736,763$ & $6,786,138$ & $2,313,513$ & $35,498,715$ \\
\hline \multirow{7}{*}{$\begin{array}{l}2008- \\
2009\end{array}$} & Pozos & & 341,268 & 19,079 & $1,882,692$ & 538,000 & - & $2,781,039$ \\
\hline & $\begin{array}{l}\text { Plantas } \\
\text { bombeo }\end{array}$ & de & - & - & - & 140,000 & - & 140,000 \\
\hline & $\begin{array}{l}\text { Red } \\
\text { distribución }\end{array}$ & de & $3,084,776$ & $2,309,718$ & $4,455,028$ & $1,666,515$ & 931,419 & $12,447,456$ \\
\hline & Red de drenaje & & $3,112,046$ & $2,509,944$ & $1,416,504$ & $3,919,995$ & $1,198,497$ & $12,156,986$ \\
\hline & Red de caminos & & $2,379,585$ & $1,722,311$ & 112,926 & 848,337 & $2,123,106$ & $7,186,265$ \\
\hline & Estructuras & & 479,987 & 330,000 & $1,771,356$ & 338,850 & 269,718 & $3,189,911$ \\
\hline & Suma de obra & & $9,397,662$ & $6,891,053$ & $9,638,506$ & $7,311,696$ & $4,522,740$ & $37,761,657$ \\
\hline \multirow{7}{*}{$\begin{array}{l}2009- \\
2010\end{array}$} & Pozos & & 551,506 & - & $2,269,957$ & 348,002 & - & $3,169,464$ \\
\hline & $\begin{array}{l}\text { Plantas } \\
\text { bombeo }\end{array}$ & de & - & - & - & 40,000 & - & 40,000 \\
\hline & $\begin{array}{l}\text { Red } \\
\text { distribución }\end{array}$ & de & $3,201,670$ & $2,502,287$ & $3,336,584$ & $2,346,631$ & 868,757 & $12,255,928$ \\
\hline & Red de drenaje & & $3,194,716$ & $3,218,245$ & $1,553,441$ & $2,671,542$ & 788,163 & $11,426,107$ \\
\hline & Red de caminos & & $2,491,327$ & $1,578,433$ & 116,313 & $2,685,922$ & $2,343,359$ & $9,215,354$ \\
\hline & Estructuras & & 699,906 & - & $3,063,265$ & 238,850 & - & $4,002,020$ \\
\hline & Suma de obra & & $10,139,124$ & $7,298,965$ & $10,339,560$ & $8,290,947$ & $4,000,278$ & $40,068,874$ \\
\hline
\end{tabular}

Fuente: elaboración propia con datos obtenidos de la CONAGUA 2007b, 2008b, 2009c. 
Cuadro 3. Comparativo entre los gastos de conservación y superficie sembrada en los módulos del DR 063

\begin{tabular}{|c|c|c|c|c|c|c|}
\hline \multirow[b]{3}{*}{ Módulos } & \multicolumn{6}{|c|}{ CICLO AGRÍCOLA } \\
\hline & \multirow{2}{*}{$\begin{array}{l}2007-2008 \\
\text { Ha. } \\
\text { Sembrada } \\
\text { s }\end{array}$} & \multirow[b]{2}{*}{$\begin{array}{l}\text { Costo de } \\
\text { conservación }\end{array}$} & \multirow{2}{*}{$\begin{array}{l}2008-2009 \\
\text { Ha. } \\
\text { Sembrada } \\
\text { S }\end{array}$} & \multirow[b]{2}{*}{$\begin{array}{l}\text { Costo de } \\
\text { conservación }\end{array}$} & \multirow{2}{*}{$\begin{array}{l}\text { 2009-2010 } \\
\text { Ha. } \\
\text { Sembradas }\end{array}$} & \multirow[b]{2}{*}{$\begin{array}{l}\text { Costo de } \\
\text { conservación }\end{array}$} \\
\hline & & & & & & \\
\hline Bamoa I-1 & 30,514 & $8,797,647$ & 30,514 & $9,397,662$ & 30,514 & $10,139,124$ \\
\hline Milpas I-2 & 23,620 & $7,864,654$ & 21,252 & $6,891,053$ & 23,623 & $7,298,965$ \\
\hline Petatlán II-1 & 22,794 & $9,736,763$ & 23,285 & $9,638,506$ & 23,285 & $10,339,560$ \\
\hline \multicolumn{7}{|l|}{ Tetameche } \\
\hline II-2 & 21,148 & $6,786,138$ & 22,281 & $7,311,696$ & 21,790 & $8,290,947$ \\
\hline Sabinal III-1 & 5,130 & $2,313,513$ & 10,040 & $5,472,740$ & 9,040 & $4,000,278$ \\
\hline Distrito 063 & 103,205 & $35,498,715$ & 107,372 & $38,711,657$ & 108,252 & $40,068,874$ \\
\hline
\end{tabular}

Fuente: elaboración propia con datos obtenidos en CONAGUA 2007b, 2008b, 2009c.

la infraestructura hidráulica del DR063, fue de $9.05 \%$ del ciclo $2007-2008$ al ciclo 2008 2009, y de un 3.5\% del ciclo 2008-2009 al ciclo 2009-2010. Este comportamiento en el presupuesto varía en función alas necesidades de los módulos de riego y los proyectos programados por sus representantes. Aun así, es importante destacar la inversión que se destina en el distrito en materia de conservación (ver Figura 4), puesto que en este rubro se erogan un promedio de 350 a 370 pesos por hectárea, destacando los módulos Petatlán II-1 y Sabinal III-1 cuya inversión por hectárea, en materia de conservación, superan los 400 pesos. Caso contrario, el módulo Milpas I-2, donde la inversión en materia de conservación por hectárea ha disminuido en los dos últimos ciclos agrícolas (Fig. 4).

En el cuadro 4, se observan los porcentajes de la aplicación de los recursos para el ciclo 2009-2010, en los distintos módulos del DR063, donde se aprecia que la asignación de dicho recurso se realiza en base a las necesidades de cada módulo, observándose que dentro de las prioridades se ubican las redes de distribución y drenaje, y las áreas estratégicas en la conservación de los recursos hídricos; esto debido a que son los conceptos que más porcentaje del gasto de conservación tienen, con un $30.59 \%$ y un $28.52 \%$, respectivamente. A nivel distrital, el $23 \%$ se destina a red de caminos, un $9.99 \%$ a estructuras y un $7.91 \%$ a pozos; en este último rubro, destaca la inversión del $21.95 \%$ de su presupuesto que se realiza en el

Figura 4. Comportamiento de las inversiones en materia de conservación por hectárea sembrada en los distintos módulos del DR063.

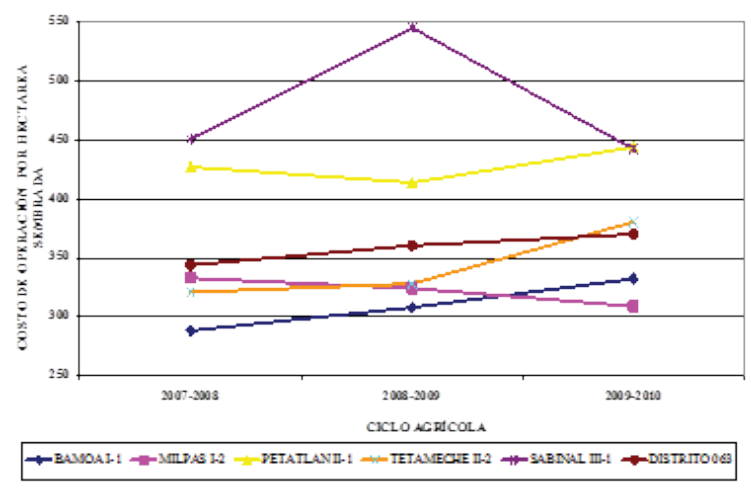

Fuente: elaboración propia con datos obtenidos en CONAGUA 2007b, 2008b, 2009c. 
Cuadro 4. Distribución porcentual de los gastos de conservación en los distintos módulos del distrito 063.

\begin{tabular}{|c|c|c|c|c|c|c|}
\hline \multirow[b]{2}{*}{ CONCEPTO } & \multicolumn{5}{|c|}{ PORCENTAJE DEL PRESUPUESTO POR CONCEPTO } & \multirow[b]{2}{*}{ DISTRITO } \\
\hline & $\begin{array}{l}\text { BAMOA } \\
\text { I-1 }\end{array}$ & $\begin{array}{l}\text { MILPA } \\
\text { S I-2 }\end{array}$ & $\begin{array}{l}\text { PETATLA } \\
\mathrm{N} \text { II-1 }\end{array}$ & $\begin{array}{l}\text { TETAMEC } \\
\text { HE II-2 }\end{array}$ & $\begin{array}{l}\text { SABIN } \\
\text { AL III-1 }\end{array}$ & \\
\hline POZOS & $5.44 \%$ & $0.00 \%$ & $21.95 \%$ & $4.20 \%$ & $0.00 \%$ & $7.91 \%$ \\
\hline $\begin{array}{l}\text { PLANTAS } \\
\text { BOMBEO }\end{array}$ & $0.00 \%$ & $0.00 \%$ & $0.00 \%$ & $0.48 \%$ & $0.00 \%$ & $0.10 \%$ \\
\hline $\begin{array}{l}\text { RED } \\
\text { DISTRIBUCION }\end{array}$ & $31.58 \%$ & $34.28 \%$ & $32.27 \%$ & $28.30 \%$ & $21.72 \%$ & $30.59 \%$ \\
\hline RED DE DRENAJE & $31.51 \%$ & $44.09 \%$ & $15.02 \%$ & $32.22 \%$ & $19.70 \%$ & $28.52 \%$ \\
\hline RED DE CAMINOS & $24.57 \%$ & $21.63 \%$ & $1.12 \%$ & $32.40 \%$ & $58.58 \%$ & $23.00 \%$ \\
\hline ESTRUCTURAS & $6.90 \%$ & $0.00 \%$ & $29.63 \%$ & $2.88 \%$ & $0.00 \%$ & $9.99 \%$ \\
\hline SUMA & $100.00 \%$ & $\begin{array}{l}100.00 \\
\%\end{array}$ & $100.00 \%$ & $100.00 \%$ & $100.00 \%$ & $100.00 \%$ \\
\hline
\end{tabular}

Fuente: elaboración propia con datos obtenidos en CONAGUA $2009 \mathrm{~b}$.

módulo Petatlán II-1, por lo que requirieron del $21.95 \%$ de su presupuesto para abastecer el suministro eficiente del agua a través de la explotación de los recursos subterráneos debido a que la disponibilidad de agua superficial es limitado. Caso especial, el módulo de riego el Sabinal III-1, donde en este mismo periodo, la inversión en la red de caminos superó el 58\% del presupuesto en materia de conservación (Cuadro 4).

En la figura 5 se puede apreciar el comportamiento de los egresos para conservación en los ciclos 2007-2008, 20082009 y 2009-2010. En general, los montos se mantienen en el distrito y en la mayoría de los módulos, siendo Sabinal el que posee una mayor variación, pues para el ciclo 2007-2008 la red de drenaje era su principal prioridad, mientras que para los ciclos 20082009 y 2009-2010 la red de caminos se convirtió en su principal necesidad.

\section{CONCLUSIONES}

Mucho se ha hablado de que México, privilegiado por la zona geográfica en la que se encuentra, es un área propicia para el desarrollo de la agricultura, actividad que es fundamental para garantizar el abasto de alimentos a la sociedad y unos de los pilares

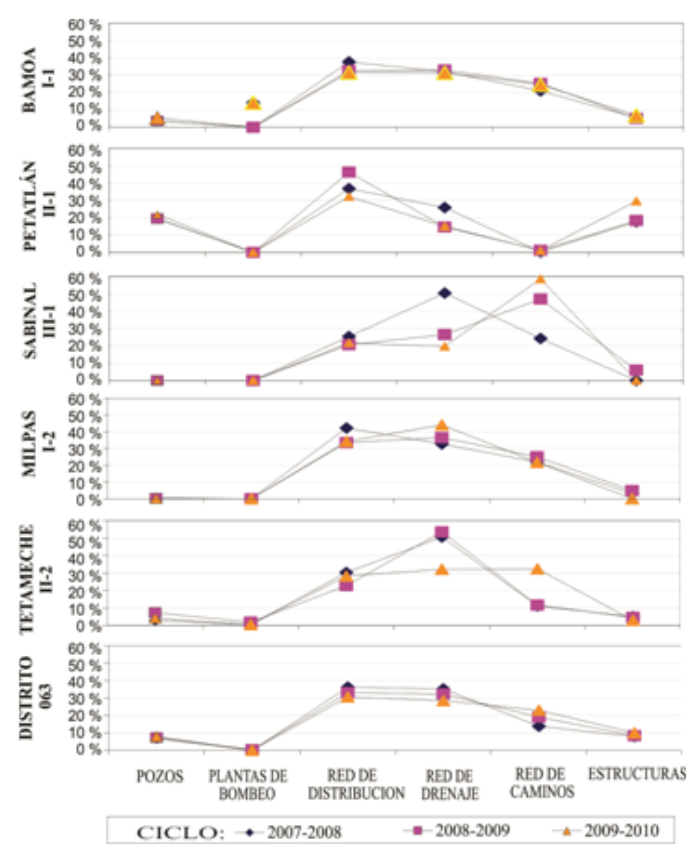

Figura 5. Análisis de los egresos en conservación por concepto y módulo de riego. Fuente: elaboración propia con datos obtenidos en CONAGUA 2007b, 2008b, 2009c 
de la economía nacional. Esta situación ha propiciado que la inversión pública y privada sea fuerte y constante en aras de la tecnificación en los procesos productivos. Este contexto demanda un incremento en la disponibilidad de agua, por lo que la inversión en la infraestructura hidráulica es fundamental para fortalecer el campo mexicano. Desafortunadamente, una serie de factores están incidiendo en el deterioro y la escasez de los recursos hídricos, que ponen de manifiesto la incapacidad gubernamental para garantizar el suministro eficiente del recurso, como lo observado en las recientes heladas presentadas en el estado de Sinaloa.

Desde esta perspectiva, es necesario generar los ambientes políticos, en el que se incentive las propuestas que fortalezcan el desarrollo técnico, administrativo $\mathrm{y}$ financiero del sector hidráulico, pero sobre todo, que se involucre la participación activa de los sectores sociales. Esto implica que los sectores gubernamentales generen más en políticas estables y eficientes, involucrándose los tres niveles de gobierno, y que se planifique a corto, mediano y largo plazo las inversiones en infraestructura que garantice el suministro del recurso, así como el control y manejo de la calidad del agua, sobre todo el de uso agrícola.

Se debe generar conciencia en todos los sectores sociales y políticos para que el agua sea considerada un bien escaso, de manera que se establezca mecanismos para reducir su desperdicio, prevenir la sobreexplotación y la contaminación de los mantos acuíferos, generando estrategias para un uso eficiente y racional de los recursos hídricos.

A raíz del proceso de transferencia de las actividades de operación, conservación, ingeniería de riego y drenaje, así como la administración de los Distritos de
Riego a los usuarios, organizados como asociaciones civiles, se generó una participación económica más activa en el uso sustentable de los suelos y el agua, además de participar económicamente en la administración, conservación y control de las aguas de uso agrícola. Esto, ha generado las condiciones para recaudar fondos económicos que permiten la inversión en la administración, conservación y operación de la infraestructura hidráulica, fomentando la competitividad en los procesos productivos en los sectores agrícolas.

No obstante, aun existen rezagos en la conservación de los recursos hídricos, puesto que, el volumen de agua que se pierde en su transporte, como lo es en el caso del DR063, es muy elevado, alcanzando niveles del $49 \%$, sin contemplar el volumen perdido por el mal manejo que se le pudiera dar dentro de las parcelas, lo que limita el suministro del líquido a mayores extensiones parcelarias que pudieran explotarse bajo un régimen de riego parcelario. Esta situación demuestra que, aun cuando las inversiones en la conservación de los recursos hídricos son significativas, existen fallas en el manejo del recurso, lo que demanda una mayor capacitación a las personas que están al frente de los módulos de riego, fomentar el desarrollo de escuelas de obras hidráulicas que permitan elevar el nivel tecnológico en la administración de los recursos hídricos. Es necesario capacitar a los productores agrícolas para el manejo sustentable del agua, para que trabajen con recetas de riego en función de la demanda del producto y los tipos de suelos, para que se regulen los trazos de riego a escalas menores implicando menos desperdicio de agua, pero sobre todo, para que se modernicen los sistemas de riego.

Es necesario a nivel gubernamental, crear 
los mecanismos para obligar a los usuarios a cuidar el recurso y tener una mayor supervisión en las inversiones realizadas por los módulos de riego, cuidando sobre todo los controles de calidad de las obras, y por último, impulsar entre la sociedad la conciencia ecológica, para que participen en los programas gubernamentales para la conservación de los mantos freáticos.

A nivel distrital y módulos de riego es necesario, impulsar el revestimiento $\mathrm{y}$ entubado de los canales que permitan incrementar la fluidez del recurso y la conservación del mismo, fortalecer la infraestructura que ya se tiene y renovar aquella que ya se encuentre fuertemente dañada; diseñar los sistemas de compuertas con niveles tecnológicos más elevados, que permitan tener más control y exactitud en los volúmenes de agua distribuidos, y gestionar a través de sus facultades administrativas mayores recursos económicos que puedan ser aplicados en la modernización de las obras hidráulicas, extendiéndolos a aquellos sectores susceptibles a las actividades agrícolas.

\section{LITERATURA CITADA}

Biswas, A. 1999. "Water Crisis: Current Perceptions and Futures Realities, en Salman ed. Groundwater: Legal and Policy Perspectivas". World Bank Technical Paper (456). Pp.1-29.

Cárdenas, Gustavo, Cárdenas J. 2009. "Agricultura, urbanización y agua". Instituto Interamericano de Cooperación para la Agricultura (IICA). San José, C.R. ISBN13: 978-92-9248-111-7, Montevideo, Uruguay. Pp. 1 - 44.

CONAGUA. 2007a. Plan de riegos Distrito 063 Guasave 2007-2008. Dirección de infraestructura hidroagrícola del Organismo de Cuenca Pacifico Norte Región III. Culiacán, México.

CONAGUA.2007b. Programadeactividades ciclo agrícola 2007-2008 para la conservación de obras de distritos de riegos transferidos. Distrito de riego 063. Subdirección general de infraestructura hidroagrícola del Organismo de Cuenca Pacifico Norte Región III. Culiacán, México.

CONAGUA. 2008a. Plan de riegos Distrito 063 Guasave 2008-2009. Dirección de infraestructura hidroagrícola del Organismo de Cuenca Pacifico Norte Región III. Culiacán, México.

CONAGUA. 2008b. Programa de actividades ciclo agrícola 20082009 para la conservación de obras de distritos de riegos transferidos. Distrito de riego 063. Subdirección general de operación del Organismo de Cuenca Pacifico Norte Región III. Culiacán, México.

CONAGUA. 2009a. Plan de riegos Distrito 063 Guasave 2009-2010. Dirección de infraestructura hidroagrícola del Organismo de Cuenca Pacifico Norte Región III. Culiacán, México.

CONAGUA. 2009b. Programa de actividades ciclo agrícola 20092010 para la conservación de obras de distritos de riegos transferidos. Distrito de riego 063. Subdirección general de operación del Organismo de Cuenca Pacifico Norte Región III. Culiacán, México.

CONAGUA. 2009c. Organismo de cuenca del pacífico norte. Portal del CONAGUA Recuperado el día 26 de mayo de 2010 en:

http://www.conagua.gob.mx/OCPn/ Es paniol/ T mp Contenido. 
aspx $\mathrm{id}=\mathrm{c} 813 \mathrm{f07c}-448 \mathrm{c}-40 \mathrm{f} 1-987 \mathrm{e}-$

$1 \mathrm{da} 36 \mathrm{~b} 9 \mathrm{~d} 14 \mathrm{~cd} \% 7 \mathrm{CCon} \% \mathrm{C} 3 \% \mathrm{~B} 3 \mathrm{c}$

enos $\% 7 \mathrm{C} 1 \% 7 \mathrm{C} 0 \% 7 \mathrm{C} 0 \% 7 \mathrm{C} 0 \% 7 \mathrm{C} 0$

CONAGUA. 2010a. Plan de riegos Distrito 063 Guasave 2010-2011. Dirección de infraestructura hidroagrícola del Organismo de Cuenca Pacifico Norte Región III. Culiacán, México.

CONAGUA. 2010b. Estadísticas del Agua en México, edición 2010. Secretaría de Medio Ambiente y Recursos Naturales. México, D.F.

De la Lanza E. G. y García C. J. L. 2002. Lagos y presas de México. Editorial AGT S.A. 2da. Edición

González-Márquez L.C. y Hansen A.M. 2009. Adsorción y mineralización de atrazina y relación con parámetros de suelos del DR 063 Guasave, Sinaloa. Rev. Mex. Cienc. Geol. 26, 587-599.

Instituto Nacional de Estadística y Geografía INEGI. 2011. Mapa de agricultura y vegetación. Sinaloa. Recuperado el día 25 de julio de 2011 en: http://mapserver.inegi.org. $\mathrm{mx} /$ geografia/espanol/estados/sin/ agri.cfm? $=444 \& \mathrm{e}=04$

Klohn, Wulf y Appelgren Bo. 1999. “Agua y agricultura". Revista CIDOB d'AFERS Internacionals. Núm 4546. Pp. 105 - 126.

PND. 2007. Plan Nacional de Desarrollo 2007 - 2012, Poder Ejecutivo Federal, 2007, México.

PNH. 2008. Programa Nacional Hídrico 2007 - 2012, Comisión Nacional del Agua, 2008, México.

Sánchez, Astello M., Arteaga Tovar, Reyes Ramírez, Arana Muñoz. 2001. Diagnostico del Módulo de Riego número 7 de la Venta-Unión Hidalgo, Oaxaca, México. Agricultura
Técnica en México, enero-junio, año/vol. 27, núm. 001, Texcoco, México, pp. 35-45

Víctor Manuel Peinado Guevara, Escuela de Ciencias Económicas y Administrativas ECEA-UAS, Carretera a las Glorias, Guasave, Sinaloa, México, correo electrónico: v_peinado@hotmail.com.

Carmen Camacho Castro, Facultad de Contaduría y Administración, FCA-UAS, Ciudad Universitaria, Culiacán, Sin. México, correo electrónico:mcccc@hotmail.com.

Deyanira Bernal Dominguez, Facultad de Contaduría y Administración, FCA-UAS, Ciudad Universitaria, Culiacán, Sin. México, deyanira2673@hotmail.com

Omar Delgado Rodríguez, Instituto Mexicano del Petróleo, Eje Central Lázaro Cárdenas Norte 152, San Bartolo Atepehuacan, Gustavo A. Madero, 07730, Distrito Federal, correo electrónico: odelgado@imp. $\mathrm{mx}$.

Héctor José Peinado Guevara, Escuela de Ciencias Económicas y Administrativas ECEA-UAS, Carretera a las Glorias, Guasave, Sinaloa, México, hpeinado75@ hotmail.com. 